\title{
Culturing and environmental DNA sequencing uncover hidden kinetoplastid biodiversity and a major marine clade within ancestrally freshwater Neobodo designis
}

\author{
Sophie von der Heydent and Thomas Cavalier-Smith \\ Department of Zoology, University of Oxford, South Parks Road, Oxford OX1 3PS, UK
}

Correspondence

Thomas Cavalier-Smith

tom.cavalier-smith@zoo.ox.ac.uk

\section{INTRODUCTION}

Kinetoplastea comprise the mainly free-living bodonid flagellates and parasitic trypanosomatids within the phylum Euglenozoa (Cavalier-Smith, 1981, 2003a, b). Bodonids are common, heterotrophic protozoa that occur in a wide variety of environments, including marine, freshwater and soil, from the tropics to the Arctic (Al-Qassab et al., 2002; Larsen \& Patterson, 1990; Patterson \& Simpson, 1996; Vørs,

Published online ahead of print on 1 July 2005 as DOI 10.1099/ ijs.0.63606-0.

tPresent address: Evolutionary Genomics Group, Department of Botany and Zoology, University of Stellenbosch, Private Bag X1, Matieland, Stellenbosch, 7602, South Africa.

Abbreviations: GTR, general time-reversible; ML, maximum likelihood; $\mathrm{PP}$, posterior probability.

The GenBank/EMBL/DDBJ accession numbers for $18 \mathrm{~S}$ rRNA gene sequences are AY753597-AY753632 (cultured isolates), AY753946-AY753984 (kinetoplastid environmental), AY749551AY749595 (freshwater neobodonid environmental) and AY749596AY749598 (marine neobodonid environmental).
1992, 1993). The bodonids Bodo saltans, Neobodo designis and Rhynchomonas nasuta are among the 20 most commonly seen zooflagellates (Patterson \& Lee, 2000); it is often suggested that such protists are cosmopolitan and may have wide ecological tolerances (Atkins et al., 1998, 2000; Finlay, 2002; Patterson \& Lee, 2000; Patterson \& Simpson, 1996). This frequent assumption of broad physiological tolerance and easy interchange between marine and freshwater environments was recently clearly contradicted for members of the genus Goniomonas, heterotrophic cryptomonads with deeply divergent marine and freshwater clades (von der Heyden et al., 2004a). In bodonids, recent evidence for $N$. designis suggested marked divergence between freshwater and marine strains, and all bodonid morphospecies, especially $B$. saltans, exhibited extensive genetic diversity (von der Heyden et al., 2004b). Despite considerable interest in free-living bodonids (Callahan et al., 2002; Doležel et al., 2000; Simpson et al., 2002), their true biodiversity has probably been grossly underestimated by simple light microscopy, as most 'species' are harder to tell apart by using light microscopy than the easily identifiable $B$. saltans (von der Heyden et al., 2004b). 
Environmental surveys using general eukaryote $18 \mathrm{~S}$ rRNA gene primers revealed a larger-than-expected diversity of free-living eukaryotes (Moon-van der Staay et al., 2001; Moreira \& López-García, 2002) but, curiously, found very few kinetoplastids - only seven environmental sequences were detected (Atkins et al., 2000; López-García et al., 2003; van Hannen et al., 1999). Rasmussen et al. (2001) used kinetoplastid-specific primers to amplify $24 \mathrm{~S} \alpha$-subunit rRNA genes from Danish lake sediments and found 27 of 43 clones sequenced to be different from each other and from known cultures, indicating a much higher diversity of kinetoplastids than realized previously. This suggests, as does the even greater diversity found recently for Cercozoa by using phylum-specific primers (Bass \& Cavalier-Smith, 2004), that group-specific primers may be better for elucidating the biodiversity of specific groups than generalized eukaryotic ones. Given the much larger database for $18 \mathrm{~S}$ than for 24S rRNA genes, culture-independent studies of kinetoplastid diversity using group-specific $18 \mathrm{~S}$ rRNA gene primers should allow more comprehensive assessment of their global diversity. To achieve this and to study their distribution among different geographical areas and environments, we here use class-specific kinetoplastid PCR primers to amplify $18 \mathrm{~S}$ rRNA genes from DNA extracted from a wide range of samples (16 environmental libraries yielded 39 new sequences). We also isolated 26 strains corresponding to the accounts of Skuja (1948) and Eyden (1977) of $N$. designis from both marine and freshwater environments in order to test whether earlier indications of divergence among strains from these contrasting environments (von der Heyden et al., 2004b) are real or an artefact of undersampling. To further test ubiquitous dispersal (Finlay, 2002) and environmental specialization within $N$. designis, we designed clade-specific PCR primers to amplify a highly variable region of the $18 \mathrm{~S}$ rRNA gene from two subclades within the $N$. designis species complex, one putatively exclusively freshwater and one marine.

Our results show that most environmental sequences fall within well-characterized groups within the free-living metakinetoplastids (Bodonida sensu stricto) and that four sequences belong to the early diverging prokinetoplastids [for the recent important distinction between prokinetoplastids and metakinetoplastids and the segregation of prokinetoplastids from 'core bodonids' as the novel order Prokinetoplastida, see Moreira et al. (2004)]. In the present paper, we shall refer collectively to all non-trypanosomatid metakinetoplastids simply as bodonids, rather than the more cumbersome term 'core bodonids' used previously (von der Heyden et al., 2004b), as we prefer to retain the order Bodonida following the removal of prokinetoplastids, with three suborders: Eubodonina (eubodonids: B. saltans), Parabodonina (parabodonids, e.g. the genus Parabodo) and Neobodonina (neobodonids). Within the $N$. designis morphospecies, we show that strains isolated from different environments fall into exclusively marine and freshwater lineages - they are not distributed randomly between environments (as sometimes assumed; Finlay, 2002;
Patterson \& Lee, 2000) - and that N. designis is probably a vast complex of at present morphologically indistinguishable, but genetically diverse, 'species'.

\section{METHODS}

Isolation, genomic DNA extraction, PCR amplification and sequencing of kinetoplastid 18S rRNA genes. Details of strains used, their sources and GenBank accession numbers are given in Table 1; Table 2 shows the sources for environmental libraries and the number of different genotypes isolated from each. All bodonid strains were isolated and their DNA was extracted, PCR-amplified and sequenced as described previously (von der Heyden et al., 2004b). Collection and processing of environmental samples were as described by Bass \& Cavalier-Smith (2004). Samples were stored at $4{ }^{\circ} \mathrm{C}$ immediately after collection and were used within a few hours of collecting, except for samples collected in the South Island of New Zealand, which remained at $4{ }^{\circ} \mathrm{C}$ for up to 5 days. The $18 \mathrm{~S}$ rRNA genes from both cultures and environmental samples were PCR-amplified by using kinetoplastid-specific primers: kineto14F (5'-CTGCCAGTAGTCATATGCTTGTTTCAAGGA-3' and kineto2026R (5'-GATCCTTCTGCAGGTTCACCTACAGCT-3'). These primers yield a PCR product of approximately $1 \cdot 9-2 \cdot 2 \mathrm{~kb}$. PCR amplification of DNA extracted from environmental samples used the same PCR mixture as described previously (von der Heyden et al., 2004b) but, to reduce chimaera formation between different $18 \mathrm{~S}$ rRNA genes within a sample, a modified cycling program was used: an initial denaturation at $94^{\circ} \mathrm{C}$ for $5 \mathrm{~min}, 35$ cycles $\left(94^{\circ} \mathrm{C}\right.$ for $30 \mathrm{~s}, 69^{\circ} \mathrm{C}$ for $36 \mathrm{~s}, 72^{\circ} \mathrm{C}$ for $4.5 \mathrm{~min}$ ) and a final extension at $72{ }^{\circ} \mathrm{C}$ for $10 \mathrm{~min}$. Prior to gel purification, four PCR products were pooled for each sample amplified.

For clade-specific amplification of a highly variable region of the $18 \mathrm{~S}$ rRNA gene for two clades of the $N$. designis species complex, two primer sets were used: FWb_681F (5'-GGAGTCGGTTACGTCCCRTCCTCCGRRYCG-3') and FWb_1244R (5'-TATTCTCTTTTGGCGGGMTCAGCAAGCGAG-3') for a freshwater clade (Fig. 7; amplifies $580 \mathrm{bp}$ ) and MARa_502F (5'-CAGAGATTTTCAATGGGGGATATTTAAYG-3') and MARa_1321R (5'-GGACGTGCTGAGGATATTCCCGWTA-3') for a marine clade (Fig. 8; amplifies 780 bp). The following cycling conditions were used for the PCRs: initial denaturation at $94{ }^{\circ} \mathrm{C}$ for $3 \mathrm{~min}, 35$ cycles $\left(94^{\circ} \mathrm{C}\right.$ for $30 \mathrm{~s}, 70^{\circ} \mathrm{C}$ for $36 \mathrm{~s}, 72^{\circ} \mathrm{C}$ for $1 \mathrm{~min}$ ), final extension at $72{ }^{\circ} \mathrm{C}$ for $5 \mathrm{~min}$ (freshwater clade), or an initial denaturation at $94^{\circ} \mathrm{C}$ for $3 \mathrm{~min}, 35$ cycles $\left(94^{\circ} \mathrm{C}\right.$ for $30 \mathrm{~s}, 69^{\circ} \mathrm{C}$ for $36 \mathrm{~s}, 72^{\circ} \mathrm{C}$ for $1 \mathrm{~min} 15 \mathrm{~s}$ ), final extension at $72^{\circ} \mathrm{C}$ for 5 min (marine clade).

All fragments were gel-purified by using a GFX kit (Amersham Biosciences) and cloned into the pCR2.1 vector (Invitrogen) by using TOPO TA cloning kits (Invitrogen). Three to five clones were picked from libraries generated from cultured strains and 12-15 clones were picked for initial screening of environmental libraries; this was later reduced to eight to ten clones. All clones were partially sequenced by using the M13F primer; the sequences were aligned with each other and those of kinetoplastids from our database. Clones were edited by using BioEdit (Hall, 1999) and checked by BLAST (Altschul et al., 1990); all clones from environmental samples proved to be kinetoplastid, showing $100 \%$ amplification specificity. To eliminate sequences represented multiple times, preliminary gamma-corrected BioNJ trees were constructed. To avoid the inclusion of identical or nearidentical clones from the same library, those differing by $>10 \mathrm{nt}$ in the first 600-800 nt sequenced were chosen for complete bidirectional sequencing and phylogenetic analysis; by excluding more similar sequences, we probably underestimated the actual diversity of closely 
Table 1. New strains of $N$. designis and Dimastigella and their place of isolation

All freshwater strains were isolated and grown in soil extract with added salts medium; marine strains were grown in Plymouth Erdschreiber medium (UKNCC, 2001). FW, Freshwater; NI, North Island.

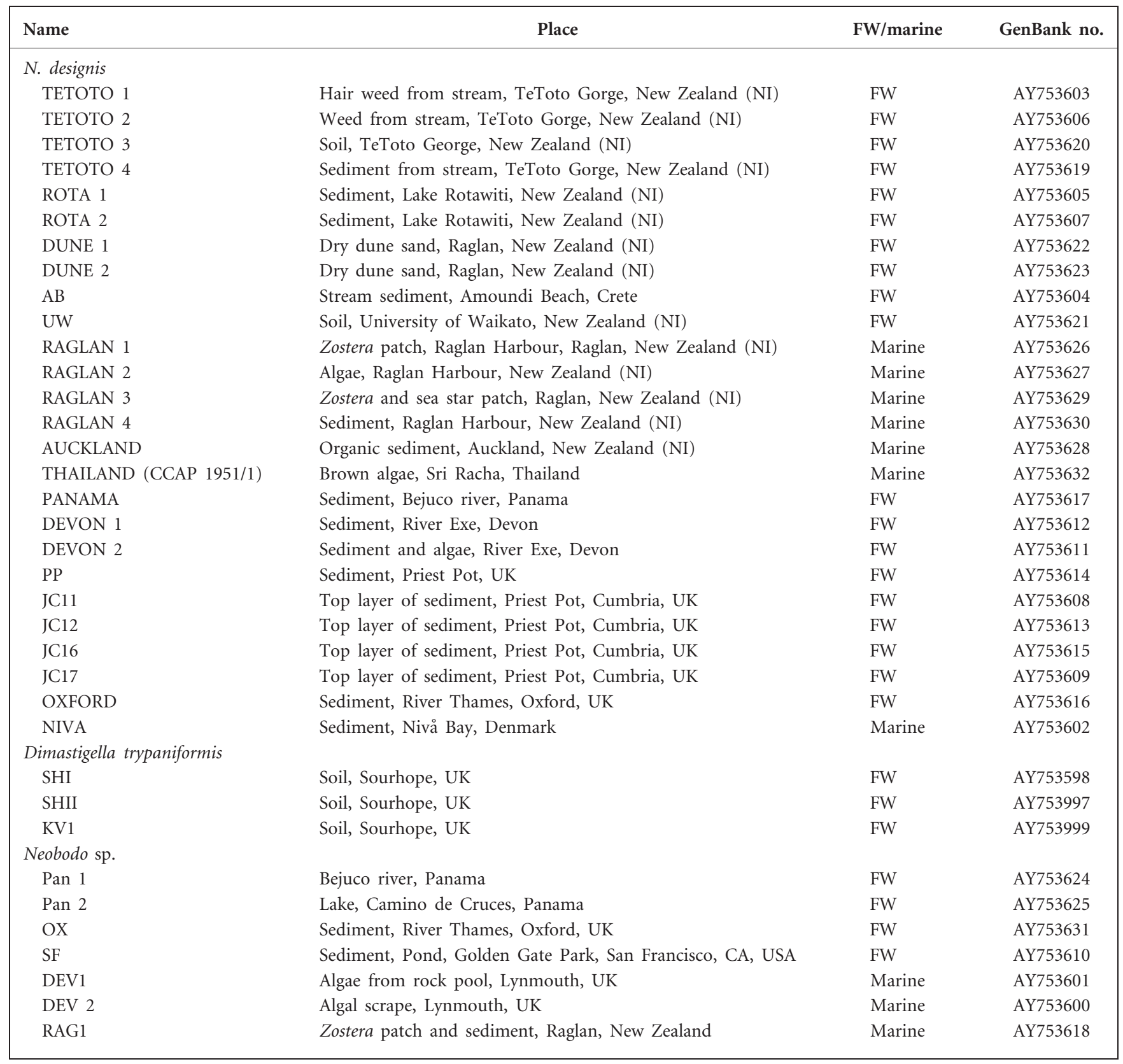

related kinetoplastid sequences. Bidirectional sequencing was carried out by using BigDye terminators with separation on an automated ABI 3730 sequencer (both from Applied Biosystems).

Sequence analysis and phylogenetic methods. Before carrying out phylogenetic analyses, all sequences were checked for chimaera formation. Firstly, the whole dataset was submitted for analysis by BELLEROPHON (Huber et al., 2004), which compares sequences within an alignment with each other to find chimaeras from separate parent sequences. Secondly, each environmental 18S rRNA gene sequence was analysed separately in three parts, each of about $710 \mathrm{nt}$, by constructing gamma-corrected neighbour-joining trees. The position of each taxon was then checked on the separate trees; additionally, each of the three sections of the 18S rRNA gene was analysed by using BLAST (Altschul et al., 1990). Chimaeras were detected easily by using these combined methods; three definite chimaeras $(7 \%)$ were found and excluded from subsequent analyses. This compares favourably with figures given by Berney et al. (2004) and Robison-Cox et al. (1995), who found 14 and $4 \cdot 1-20 \%$ of sequences to be chimaeric in analyses of eukaryote and prokaryote environmental surveys, respectively.

Analyses used PAUP* v. 4.0b10 (Swofford, 2001) with the general timereversible (GTR) substitution model. To allow for rate variation across sites, all methods assumed a gamma distribution $(\Gamma)$ and proportion of invariable sites (I), estimated by MODELTEST v. 3.06 (Posada \& 
Table 2. Environmental library description, including source and the number of different genotypes isolated from each

Note that only the most abundant genotypes will have been detected from each library; also, only clones differing in single sequencereaction tests by $>10 \mathrm{nt}$ were sequenced fully - this probably underestimated the actual diversity of closely related sequences in each sample. FW, Freshwater; NI, North Island; NP, National Park; SI, South Island.

\begin{tabular}{|c|c|c|c|}
\hline Primers/library name & Source & FW/Marine & No. genotypes \\
\hline \multicolumn{4}{|c|}{ Kineto $14 \mathrm{~F}+$ Kineto2026R } \\
\hline L1 & Muddy sand and diatom film, Vancouver, BC, Canada & Marine & 1 \\
\hline $\mathrm{L} 2$ & Sediment, Harrison Hot Springs, BC, Canada & FW & 2 \\
\hline L3 & Muddy ditch by road, Capira, Panama & FW & 1 \\
\hline L4 & Submerged stones, Bejuco River, Panama & FW & 1 \\
\hline L5 & Sediment/algae from lake, Camino de Cruces, NP Panama & FW & 4 \\
\hline L6 & Leaf litter in rainforest, Cerro Campana NP, Panama & FW & 3 \\
\hline L7 & Sediment/algae from very eutrophic lake, Gamboa, Panama & FW & 1 \\
\hline L8 & Scrape from rocks, edge of sea, nr Portobelo, Panama & Marine & 5 \\
\hline L9 & Algae from river, Wilderness, Western Cape, South Africa & FW & 1 \\
\hline L10 & Sediment and water, Sakhalin island, Russia & FW & 3 \\
\hline L11 & Algae and mud, Okavango Delta, Botswana & FW & 5 \\
\hline L12 & Moss on tree, Rotorua, New Zealand (NI) & FW & 3 \\
\hline L13 & Algae and water, Harris Tarn, New Zealand (SI) & FW & 3 \\
\hline L14 & Algae and water, Raglan, New Zealand (NI) & Marine & 1 \\
\hline L15 & Stream, Amoundi Beach, Crete & FW & 2 \\
\hline L16 & Sediment, Priest Pot, Cumbria, UK & FW & 3 \\
\hline \multicolumn{4}{|c|}{ FWb_681F+FWb_1244R } \\
\hline FW1 & Loamy soil, Anaura Bay, New Zealand (NI) & FW & 1 \\
\hline FW2 & Moss, Anaura Bay, New Zealand (NI) & FW & 3 \\
\hline FW3 & Sediment, small tarn on Harris Saddle, New Zealand (SI) & FW & 3 \\
\hline FW4 & Sediment, small tarn on Harris Saddle, New Zealand (SI) & FW & 2 \\
\hline FW5 & Stream, nr TeToto Gorge, New Zealand (NI) & FW & 1 \\
\hline FW6 & Stones and sediment, Hamilton Lake, New Zealand (NI) & FW & 3 \\
\hline FW7 & Water column, Hamilton Lake, New Zealand (NI) & FW & 2 \\
\hline FW8 & Soil, Singapore Botanic Gardens, Singapore & FW & 1 \\
\hline FW9 & Sediment and algae, Priest Pot, Cumbria, UK & FW & 3 \\
\hline FW10 & Sediment, Priest Pot, Cumbria, UK & FW & 2 \\
\hline FW11 & $1.5 \mathrm{~m}$ depth water column, Priest Pot, Cumbria, UK & FW & 2 \\
\hline FW12 & $2 \cdot 0 \mathrm{~m}$ depth water column, Priest Pot, Cumbria, UK & FW & 3 \\
\hline FW13 & Sediment, Grasmere Lake, Cumbria, UK & FW & 1 \\
\hline FW14 & Sediment and algae, Grasmere Lake, Cumbria, UK & FW & 1 \\
\hline FW15 & Moss, Buttermere Lake, Cumbria, UK & FW & 1 \\
\hline FW16 & Eutrophic pond next to Panama canal, Gamboa, Panama & FW & 1 \\
\hline FW17 & Sediment and rock scrapings, Bejuco river, Panama & FW & 1 \\
\hline FW18 & Sediment and rock scrapings, Bejuco river, Panama & FW & 5 \\
\hline FW19 & Sediment, Harrison Hot Springs, BC, Canada & FW & 2 \\
\hline FW20 & Freshwater stream, Amoundi Beach, Crete & FW & 4 \\
\hline \multicolumn{4}{|c|}{ MARa_502F+MARa_1321R } \\
\hline M1.1 & Scrapings from coastal rocks, nr Portobelo, Panama & Marine & 1 \\
\hline M2.3 & Scrapings from coastal rocks, nr Portobelo, Panama & Marine & 1 \\
\hline M3.4 & Sediment and algae, Raglan, New Zealand & Marine & 1 \\
\hline
\end{tabular}

Crandall, 1998). All heuristic analyses used tree bisection-reconnection for branch rearrangement. Dataset 1 [50 taxa, 1326 positions; prokinetoplastids and metakinetoplastids, using two euglenozoan environmental sequences as outgroup; the closest outgroup found in our previous analyses (von der Heyden et al., 2004b)] was analysed by using maximum likelihood $(\mathrm{ML})(\mathrm{GTR}+\mathrm{\Gamma}+\mathrm{I}$; four discrete rate categories) with a heuristic search with 10 random-addition sequence replicates; heuristic bootstrap analyses used 50 resamplings with one addition-sequence replicate. Datasets 2 (149 taxa, 1598 positions; all available core bodonids and trypanosomatids; prokinetoplastids were excluded to allow inclusion of more nucleotide positions) and 3 (76 taxa, 1720 positions; all available $N$. designis sequences and other clade 1 taxa as outgroups) were analysed by using weighted least squares and a heuristic search of 100 random stepwise-addition sequence replicates; bootstrap analyses used 500 resamplings with one addition-sequence replicate, but each replicate was restricted to 
10 min. Dataset 4 (37 taxa, 1745 positions; all available B. saltans sequences and a selection of other bodonids as outgroup) was analysed by using ML (GTR $+\Gamma+\mathrm{I}$; four discrete rate categories) with a heuristic search with 10 random-addition sequence replicates; heuristic bootstrap analyses used 75 resamplings with one addition-sequence replicate. Dataset 5 (100 taxa, 546 positions; freshwater subclade of $N$. designis) was analysed by using distance (BioNJ) methods; bootstrap analyses used 1000 BioNJ replicates. Dataset 6 (50 taxa, 728 positions; the marine subclade of $N$. designis) was analysed by using the minimum-evolution method, with a heuristic search of 50 random stepwise-addition sequence replicates; bootstrap analyses used 250 resamplings with one addition-sequence replicate. Alignments are available on request.

Light and electron microscopy of neobodonid strains. Live cells of three genetically divergent $N$. designis strains (freshwater, JC11 and Panama; marine, Thai) were examined by phase-contrast light microscopy with a $\times 100$ objective on a Zeiss Axiovert 200 microscope and photographed with a Roper Scientific Coolsnap HQ camera. For electron microscopy (carried out by Mr Ken Clarke, $\mathrm{CEH}$ Windermere, UK), live cells were fixed in $2 \%$ glutaraldehyde in $0.1 \mathrm{M}$ sodium cacodylate buffer for $60 \mathrm{~min}$ at $15^{\circ} \mathrm{C}$. Samples were centrifuged, washed in the same buffer and fixed for a further $30 \mathrm{~min}$ in $2 \%$ osmium tetroxide in $0.1 \mathrm{M}$ cacodylate buffer; samples were washed three times in $0 \cdot 1 \mathrm{M}$ cacodylate buffer and resuspended. Suspended cells $(5 \mu \mathrm{l})$ were placed onto Formvar-coated grids, dried at room temperature and negatively stained with $1 \%$ uranyl acetate in aqueous solution containing $0.001 \%$ bacitracin.

\section{RESULTS}

Our kinetoplastid-specific primers amplified the $18 \mathrm{~S}$ rRNA gene from 16 diverse environmental samples, yielding sequences from all major free-living clades within the subclass Metakinetoplastina except for parabodonids (unsurprisingly, not from any trypanosomatids or other parasites) and also from Prokinetoplastida, which suggests that there were no gross amplification artefacts amplifying any one clade preferentially (Fig. 1). Nine environmental libraries from temperate environments and seven from tropical environments were constructed (Table 2). Most

(a)

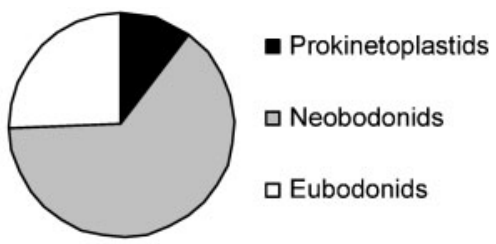

(b)

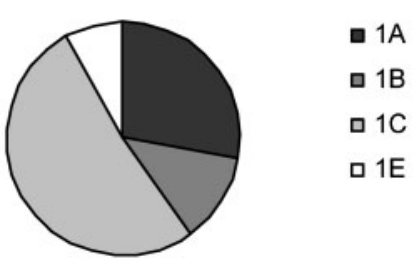

Fig. 1. Pie charts showing the percentages of environmental sequences recovered for (a) each clade of prokinetoplastids and metakinetoplastids and (b) subclades of clade 1 for which the majority of environmental sequences were found. new sequences belong to neobodonids (clade 1: Neobodo, Rhynchobodo, Rhynchomonas, Dimastigella, Cruzella, Cryptaulaxoides-like), but eubodonids (clade 3: B. saltans) are also well-represented. In total, we aligned 75 new bodonid sequences (including three Dimastigella and seven sequences from strains that group closely as sister to Cryptaulaxoides-like) to all previously published ones. There is extensive genetic diversity in a number of lineages, notably $N$. designis, B. saltans and the genus Rhynchobodo. Of the 39 different environmental sequences found, four group with the early-diverging prokinetoplastids with good support (77\%) (Fig. 2). Two of these (L4.9 and L12.4) group as sister to the Ichthyobodo/Perkinsiella symbiont clade with moderate $(66 \%)$ bootstrap support. Two other sequences (L7.7 and L8.10) group with the unknown deep-sea hydrothermal-vent environmental sequence AT4-56. All other environmental sequences generated in this study group within core bodonids: all group within clades that contain isolates of known morphology (Fig. 3). Clade 1 (neobodonids) contains the majority of new environmental sequences (64\%). Clade 3 (eubodonids) contains $26 \%$; no environmental sequences were found in clade 2 (parabodonids), which mainly consists of parasitic species. Fig. 1 shows the percentages of environmental sequences within the major clades of kinetoplastids and neobodonids. All three major clades have strong bootstrap support (Fig. 3); clade 1 (Neobodonina; $86 \%$ ), clade 2 (Parabodonina; $91 \%$ ), clade 3 (Eubodonina; $100 \%$ ). Clade 1 is further subdivided into five clades, labelled 1A-1E (von der Heyden et al., 2004b). Fig. 3 also includes sequences for three additional Dimastigella trypaniformis strains (kindly donated by Professor K. Vickerman, Glasgow, UK) and seven newly isolated strains that group as sister to Cryptaulaxoides-like in clade 1D.

We isolated 26 strains of bodonids that were indistinguishable in morphology from N. designis (Eyden, 1977; Skuja, 1948) and found that they fall into clade 1C and can be subdivided into six moderately to robustly supported major lineages (designated Nd1-Nd6; Fig. 4) that consist almost exclusively of marine or freshwater isolates and/or environmental sequences, and that, within each clade, there is remarkable genetic diversity among even closely related strains. Fig. 5 shows light micrographs of three genetically divergent strains of $N$. designis to illustrate their similarity. Fig. 6 shows electron micrographs of one marine and one freshwater $N$. designis strain, whose cilia were examined for the presence of mastigonemes.

By using still more-specific primers designed to amplify only a narrow array of genotypes within one marine and one freshwater clade (indicated by two boxes in Fig. 4), we further explored the genetic diversity of two subclades of $N$. designis. We obtained 42 new sequences from 20 environmental libraries within the freshwater clade (Table 1, Fig. 7); the freshwater-specific primers amplified products only from freshwater samples. Bootstrap support for lineages within this subclade is generally low; support for the clade itself is high (Fig. 7; $90 \%$ ). Although we screened 


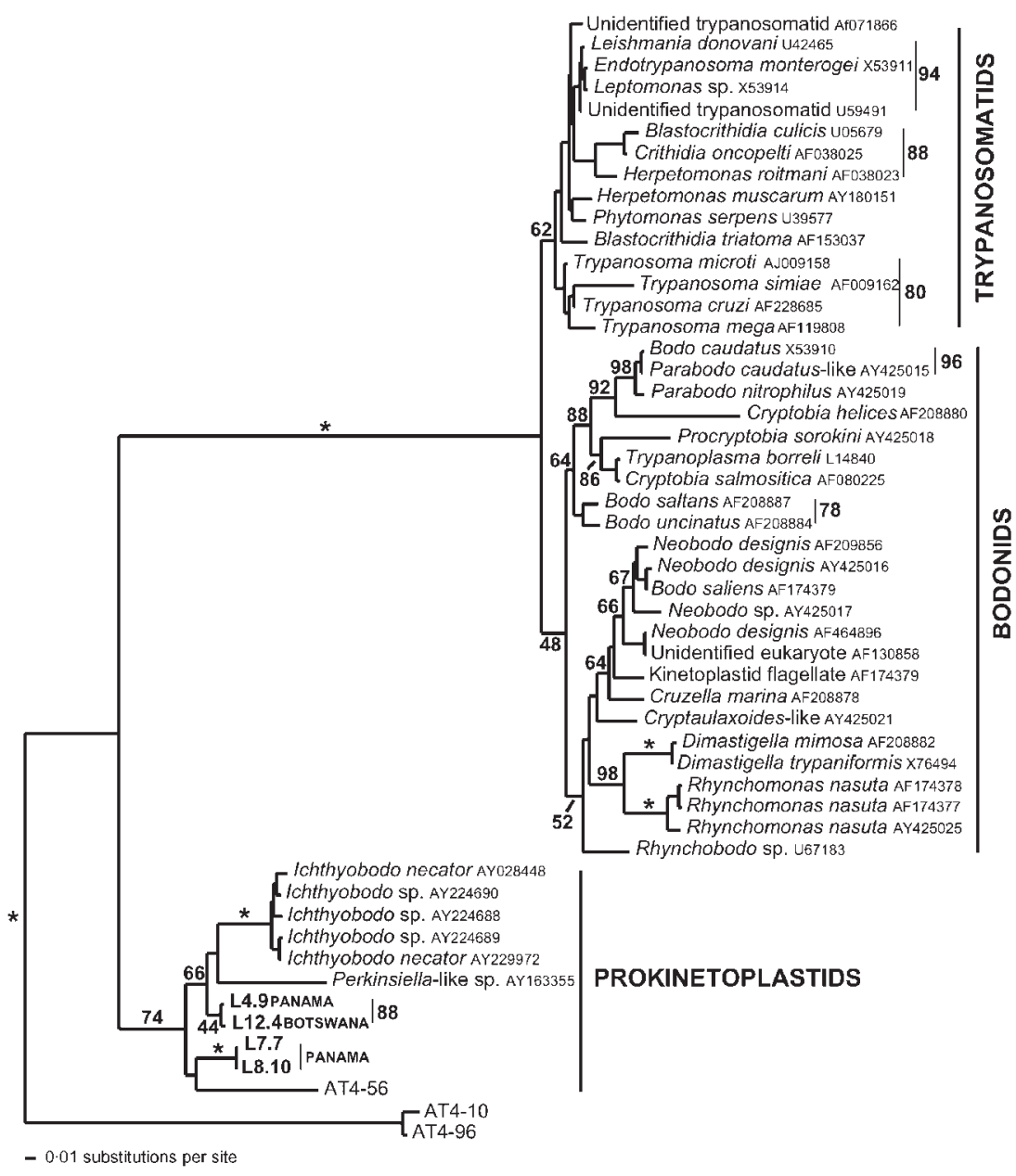

Fig. 2. $M L$ tree of $5018 \mathrm{~S}$ rRNA gene sequences of kinetoplastids and two outgroup lineages using 1326 positions $(a=0.522712$, $i=0.305469, \quad-\ln \mathrm{L}=10526 \cdot 49)$. This tree included only the new prokinetoplastid environmental sequences; the new bodonid environmental sequences are shown in Fig. 3. The tree is rooted as described previously (LópezGarcía et al., 2003; Moreira et al., 2004; von der Heyden et al., 2004b). New sequences are shown in bold. Bootstrap percentages from 50 replicates are given at major nodes; percentages $>43 \%$ are shown. Asterisks denote $100 \%$ bootstrap support.
150 DNA samples, most did not show amplified bands on agarose gels and we could construct only three libraries for the marine subclade, suggesting either that there are fewer neobodonid species in marine environments or that they are less abundant, and therefore were not recovered by using our methodology. At least four lineages can be distinguished within the marine clade, each with robust bootstrap support (Fig. 8; 95-100\%), but overall bootstrap support for this major marine clade is low (52\% in Fig. 4; $33 \%$ in Fig. 8). Two lineages contain strains exclusively from either the northern or southern hemispheres; one contains strains from both hemispheres and one is represented by only one environmental sequence.

In a separate ML analysis of $B$. saltans isolates and environmental sequences (Fig. 9), the clade of sequences from Priest Pot, Cumbria, UK (L16.16 and L16.17) did not branch as deeply as in the distance tree in Fig. 3, but within a bigger clade of northern-hemisphere sequences. An environmental sequence from Panama (L5.5) is the most-divergent eubodonid sequence.

\section{DISCUSSION}

Our results suggest that the major clades that constitute overall kinetoplastid diversity have probably now been revealed, as all new environmental sequences fall within groups for which the morphology has been at least partially characterized; there is, however, great genetic diversity within individual clades and between morphologically identical strains, suggesting that there are large numbers

Fig. 3. Distance tree of $14918 \mathrm{~S}$ rRNA gene sequences of all available bodonids and a selection of 13 trypanosomatids used as outgroup, based on 1598 positions (weighted least squares power 2; GTR $+\Gamma+$ I model; $a=0 \cdot 492434, i=0 \cdot 360997$ ). Newly sequenced taxa are shown in bold. Sequences from environmental libraries are given by the letter $L$ followed by their library designation (refer to Table 2 for library source). Bootstrap support values are from 500 replicates (restricted to 10 min per replicate); asterisks denote $100 \%$ support; where no values are given, support is $<50 \%$. Refer to von der Heyden et al. (2004b) for GenBank accession numbers for additional taxa used in these analyses. The branch length of Cryptobia helicis has been shortened by $50 \%$ in order to fit. 


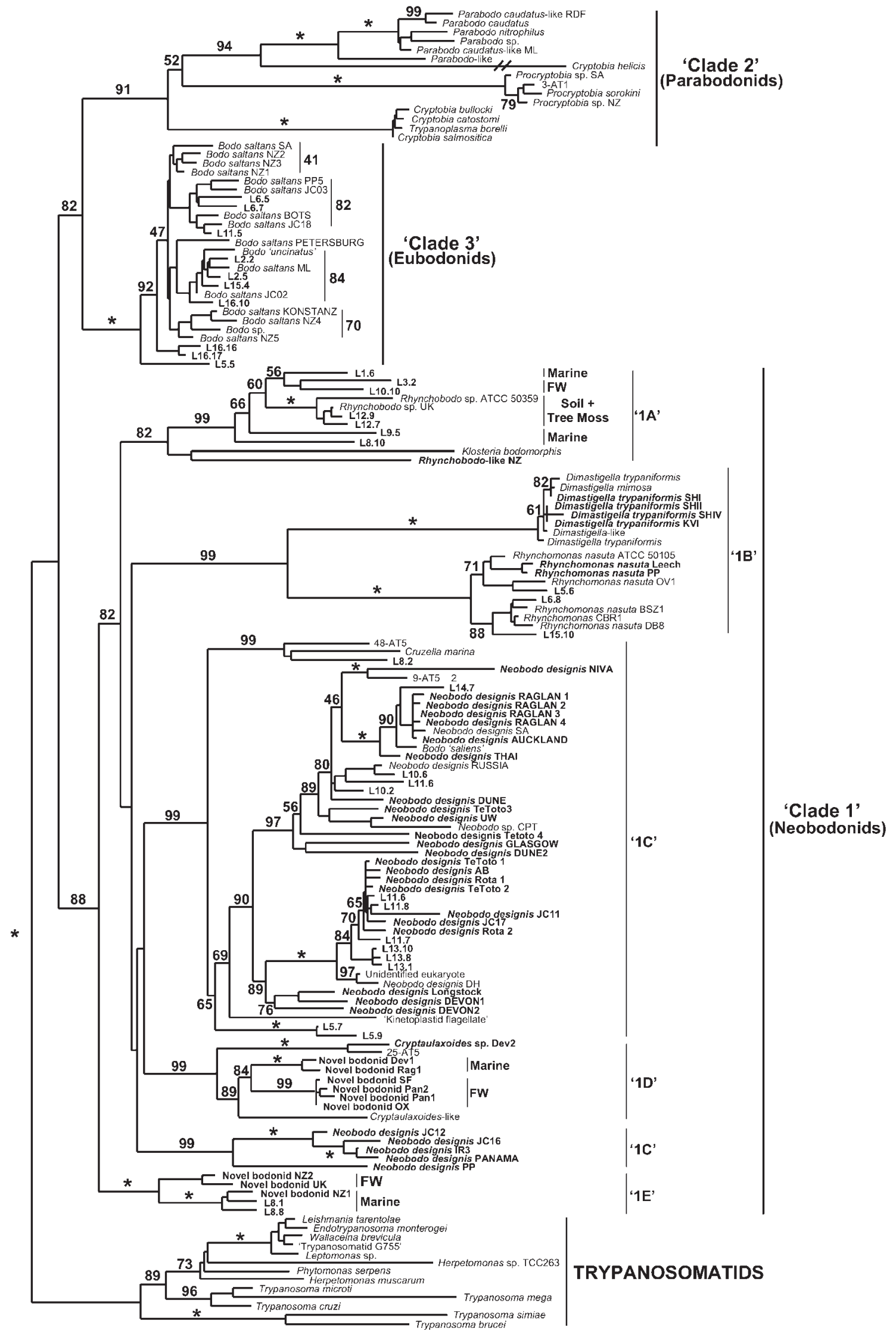

- 0.005 substitutions per site 


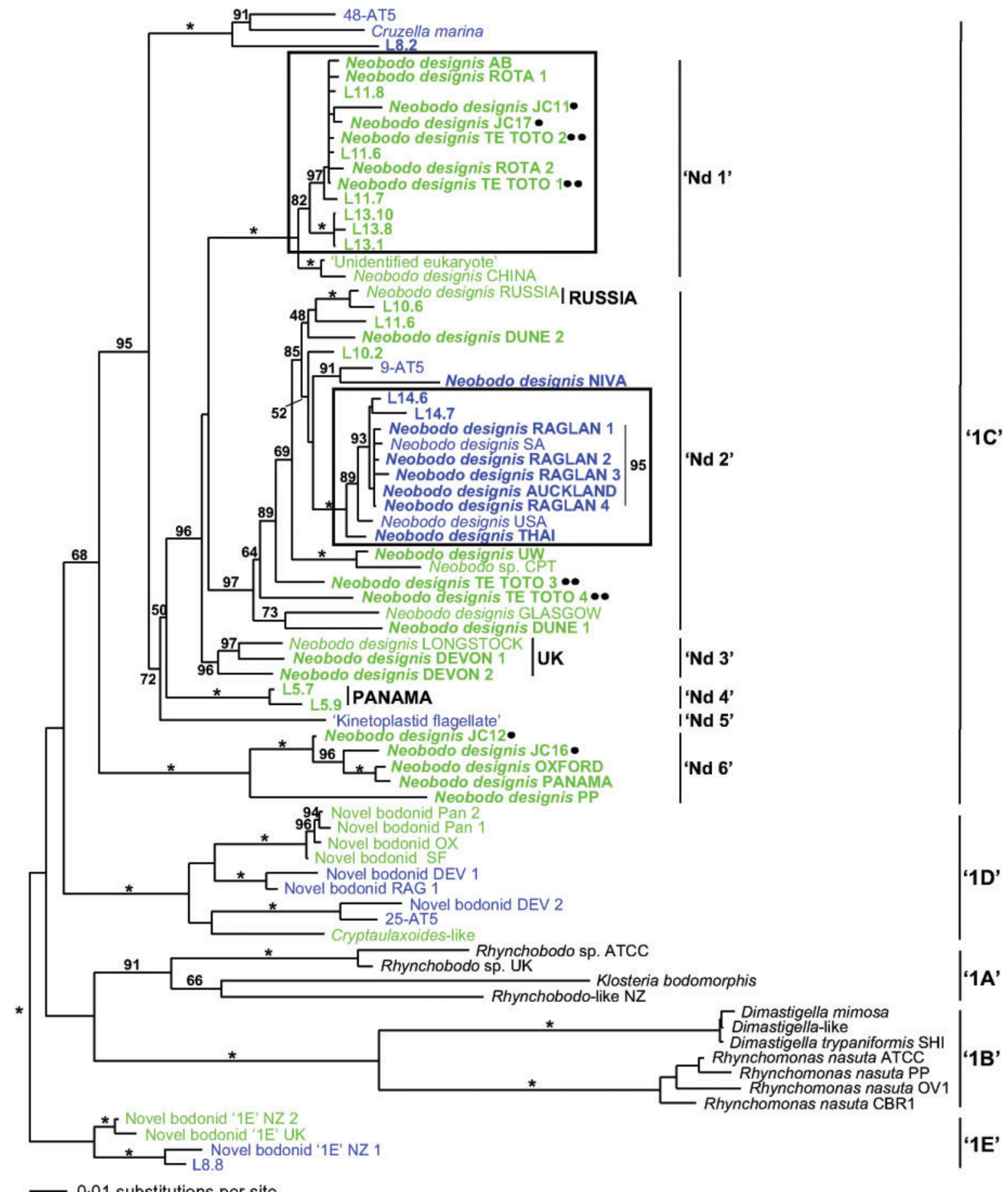

Fig. 4. Distance tree of $7618 \mathrm{~S}$ rRNA gene sequences of $N$. designis strains and other clade 1 outgroups using 1720 positions (weighted least squares power 2; GTR $+\Gamma+\mathrm{I}$ model; $a=0.554782, i=0 \cdot 444142$ ). Lineages marked in green denote sequences from freshwater; those in blue are marine. New $N$. designis sequences only are shown in bold. Bootstrap percentages from 500 replicates (restricted to $10 \mathrm{~min}$ per replicate) using the WLS2 method are shown; asterisks denote $100 \%$ support; where no values are given, support is $<48 \%$. Boxes mark the clades for which clade-specific primers were designed. Dots after lineage names refer to those strains that were collected from the same geographical place at the same time; one dot represents strains from Priest Pot, Cumbria, UK; two dots mark strains from TeToto Gorge, New Zealand. 

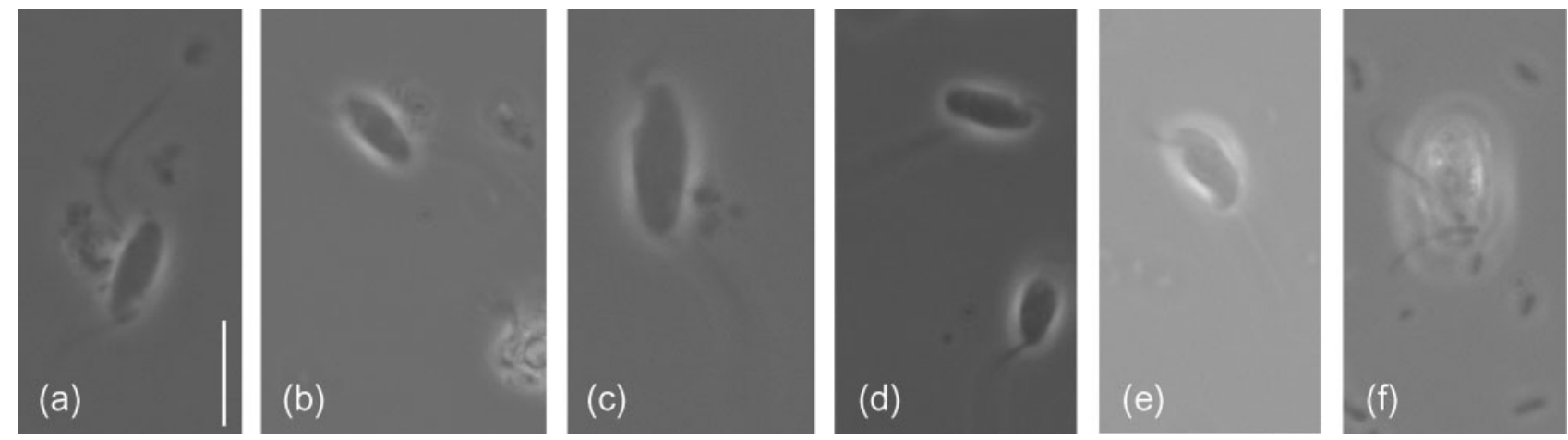

Fig. 5. Phase-contrast light micrographs of living cells of three genetically divergent strains of $N$. designis to illustrate their practical inseparability by light microscopy. (a, b) N. designis JC11 (freshwater); (c, d) N. designis Panama (freshwater); (e, f) N. designis Thai (marine). Bar, $8 \mu \mathrm{m}$.

of cryptic individual species within bodonids and prokinetoplastids and that the majority of lineages are species complexes that need a great deal more detailed study.

\section{Diversity of prokinetoplastids}

We obtained four new sequences that group within the early-diverging prokinetoplastids from samples from
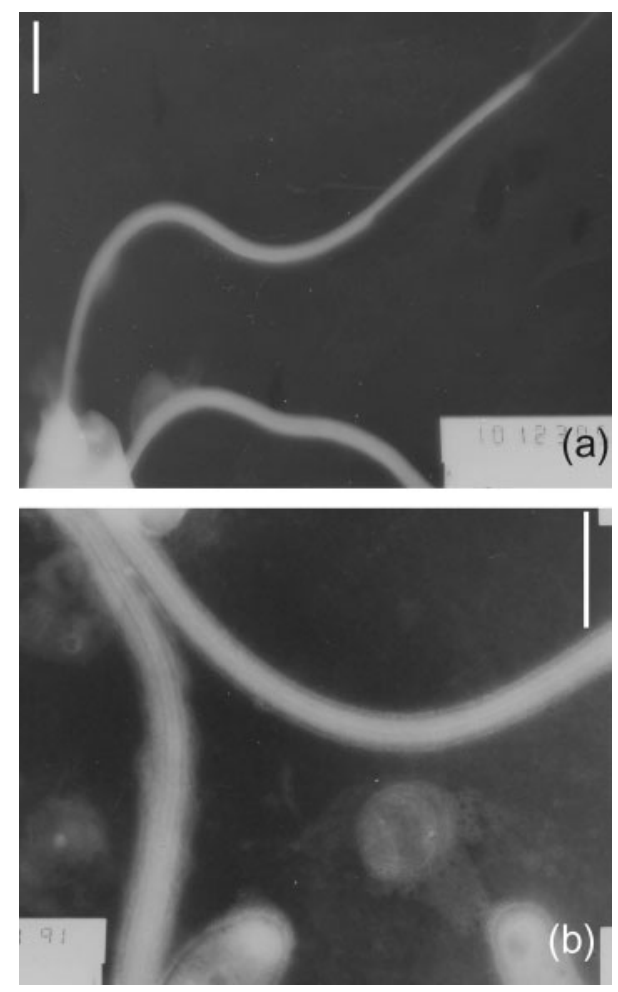

Fig. 6. Electron micrographs of two strains of $N$. designis (one marine and one freshwater) showing both the anterior and posterior cilia; no mastigonemes were found on either strain. (a) N. designis JC11 (freshwater); the acronematic tip of the posterior cilium can be seen clearly. (b) $N$. designis Thai (marine). Bars, $1 \mu \mathrm{m}$.
Panama and Botswana (Fig. 2). Two (L7.7 and L8.10) form a substantially divergent sister group to an environmental sequence from deep-sea hydrothermal-vent samples (AT4-56), which is a lineage not yet characterized by morphology. Two others (L4.9 and L12.4) from Panama and Botswana group as sister to Ichthyobodo/Perkinsiella. It is not known whether these environmental lineages represent true free-living prokinetoplastids or are dispersal phases for parasitic species such as Ichthyobodo. It is interesting to note, however, that all environmental sequences related to the parasitic prokinetoplastids have been found from warm tropical environments or deep-sea hydrothermal vents, suggesting that they may be more prolific in these conditions, perhaps because of a larger pool and broader spectrum of hosts to parasitize (Moreira \& López-García, 2003).

\section{Diversity of free-living metakinetoplastids (Bodonida sensu stricto)}

Clade 1 (neobodonids) contains the majority of sequences obtained directly from environmental samples $(64 \%)$ and the majority of these are found in clades containing isolates of Neobodo (Figs 1 and 3). Clade 3 (eubodonids) contains $26 \%$ of all recovered environmental sequences. As we obtained sequences from the two clades that contain the majority of free-living kinetoplastids, it is unlikely that this is caused by a PCR amplification bias towards them, but may indicate a genuinely greater abundance of these clades over others in environmental samples. This does not mean that other kinetoplastids (e.g. Parabodo) may not have been present in the samples, but that they are much scarcer and our methods may not have been sensitive enough to reveal them in all samples. Moreover, N. designis and B. saltans, for which we also found a high number of environmental sequences, are two of the 20 most commonly observed zooflagellates (Patterson \& Lee, 2000), so it is no surprise that they occurred most often. However, the ease of detecting these morphospecies microscopically and by culturing and environmental DNA sequencing could largely be because each comprises such a large number of different strains, rather than because any one strain is abundant or 


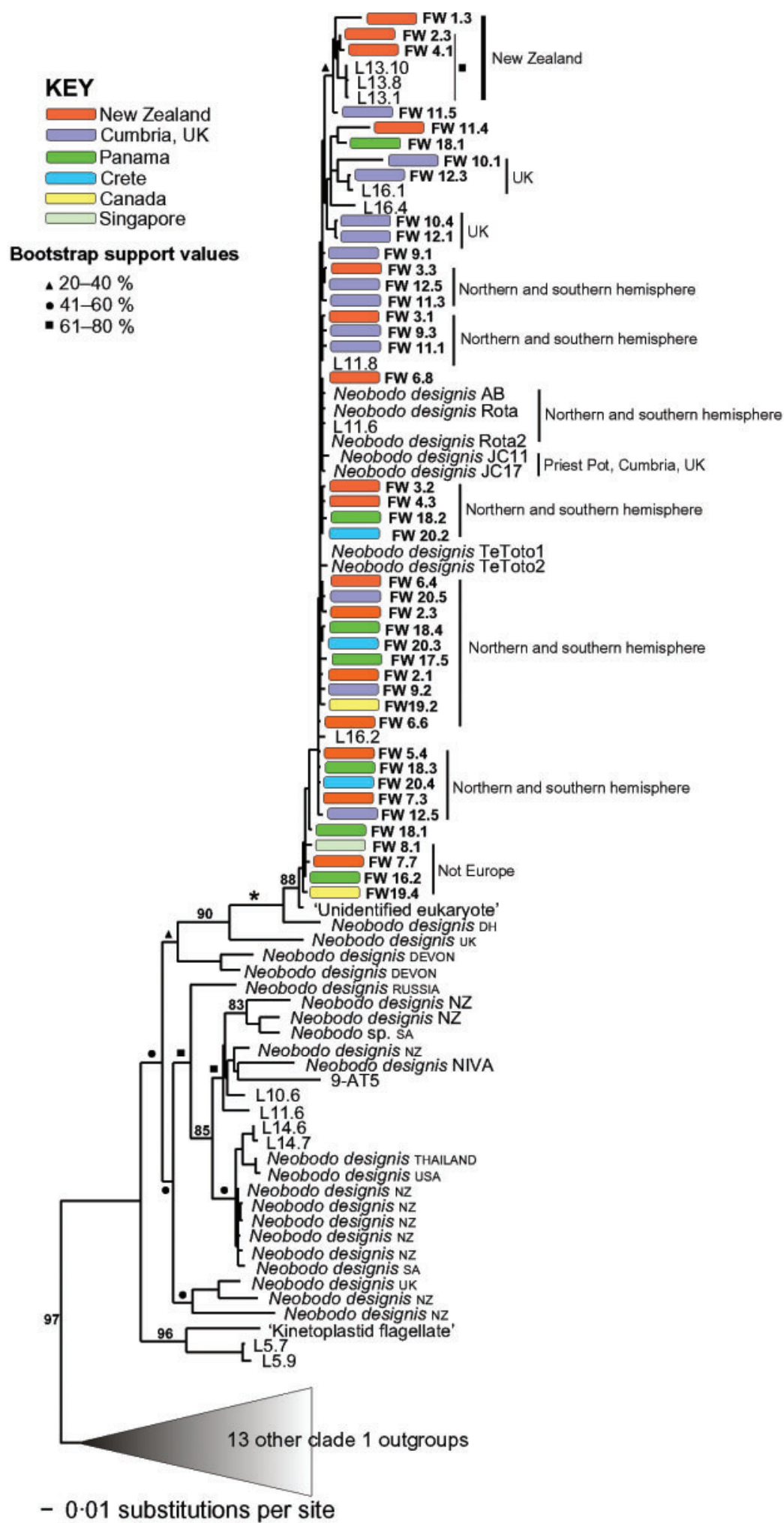

Fig. 7. Distance tree of $10018 \mathrm{~S}$ rRNA gene sequences of clade 1 neobodonids based on 546 positions (BioNJ; GTR $+\Gamma+1$ model; $a=0.743192, \quad i=0.302549)$. The individual environmental sequences are colour-coded to describe their geographical origin, as shown in the key. Bootstrap support values from 1000 BioNJ replicates $>20 \%$ are shown; symbols denoting bootstrap support values are explained in the key.

widespread. We never identified the same sequence twice from different libraries, suggesting that the total number of strains is very large.

Clade 1A (Rhynchobodo, Klosteria) contains $28 \%$ of environmental sequences, all of which group within the
Rhynchobodo lineage, indicating that members of this genus are also fairly common in the environment. Clade 1B (Dimastigella/Rhynchomonas) contains $12 \%$ of sequences found from environmental samples, all of which fall in the $R$. nasuta clade. Clade 1C (Neobodo/Cruzella) contains $52 \%$ of all environmental sequences, indicating 


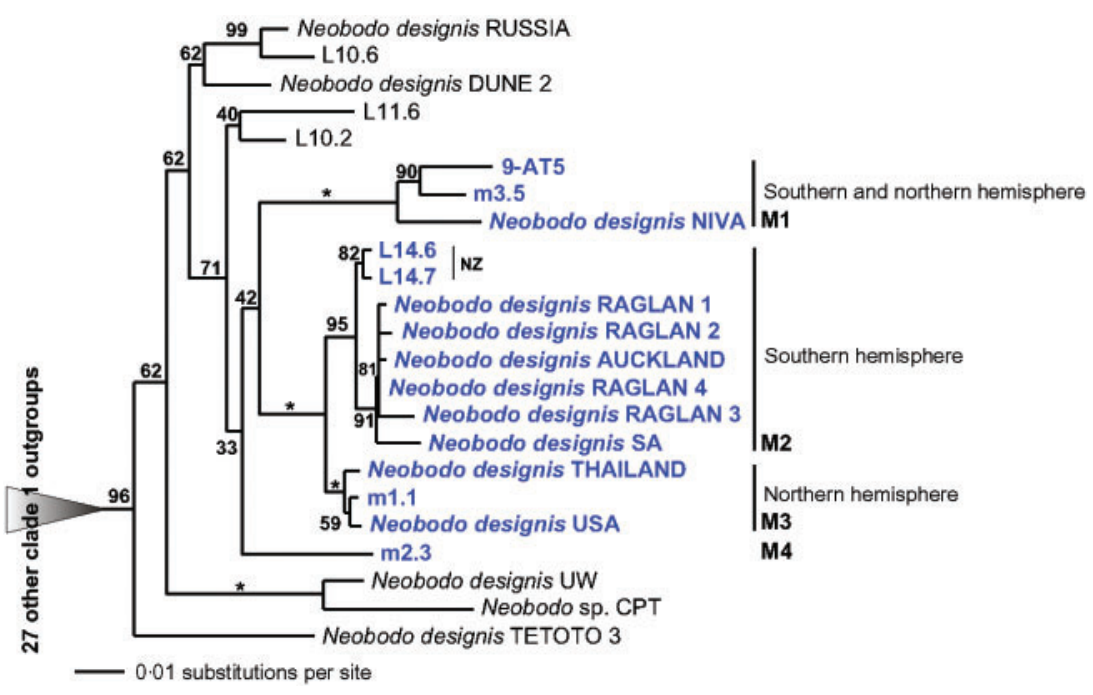

Fig. 8. Distance tree of $5018 \mathrm{~S}$ rRNA gene sequences of marine neobodonids and outgroups based on 728 positions (minimum evolution; GTR $+\Gamma+$ I model; $a=0.591899$, $i=0 \cdot 406543$ ). Bootstrap replicates are from 250 heuristic resamplings; supports $>40 \%$ are shown. The marine clade is coloured blue; clades M1-M4 and their geographical origins are shown. that representatives of this clade are the most commonly occurring of all kinetoplastids and that they are found in a

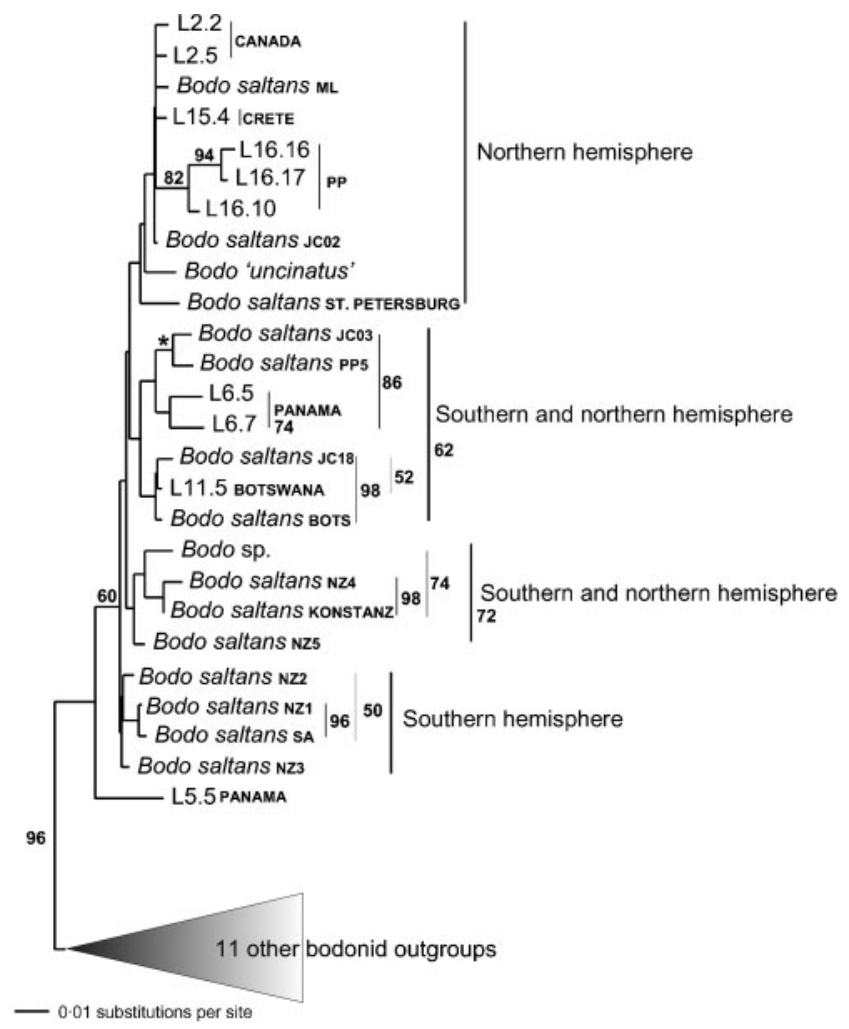

Fig. 9. $\mathrm{ML}$ tree of $3718 \mathrm{~S}$ rRNA gene sequences comprising clade 3 ( $B$. saltans species complex) using 1745 positions $(\mathrm{GTR}+\Gamma+\mathrm{I} \quad$ model; $\quad a=0.533397, \quad i=0.374946, \quad-\ln \mathrm{L}=$ 9907.62873). The tree is rooted on 11 other bodonid outgroups. Bootstrap support percentages from 75 replicates $>46 \%$ are given; an asterisk denotes $100 \%$ support. 'Bodo sp.' and ' $B$. uncinatus' are probably B. saltans (Moreira et al., 2004; von der Heyden et al., 2004b). Strain JC02 is now CCAP 1907/6. wide variety of environmental conditions. Clades 1D (novel bodonid '1D'; including Cryptaulaxoides-like) and 1E (novel bodonid lineage) contain no and two ( $8 \%$ ) environmental sequences, respectively, suggesting that they are far less common in number and/or abundance than other clades. However, our sequences of seven new cultures (six of which are sister to a clade containing Cryptaulaxoides-like) of clade $1 \mathrm{D}$ indicate for the first time that this clade is comparable in phylogenetic depth to the major Neobodo clade and, like it, has marine and freshwater subclades (Fig. 4).

We found no environmental sequences for clade 2 (parabodonids), which suggests that free-living members are quite rare, at least in our samples. Clade 3 (eubodonids) contains $26 \%$ of all environmental sequences; most of these fall into well-defined subclades within the B. saltans species complex. Sequence L5.5 from lake sediment in Panama (Table 2) is sister to all others (Figs 3 and 7) and need not necessarily have the same morphology. As the $B$. saltans species complex is studied in more detail, it will probably need to be divided into several species (Callahan et al., 2002; von der Heyden et al., 2004b) and identifying the Panama lineage might provide valuable clues as to the ancestry of the $B$. saltans species complex.

A previous study investigated kinetoplastid diversity in a Danish lake sediment by using PCR primers specific for the $24 \mathrm{~S} \alpha$-subunit of rRNA (Rasmussen et al., 2001) and found a high diversity (27 different clones of a total of 43) of kinetoplastid sequences. They found that the majority of their sequences grouped within the free-living bodonids, but also that some grouped as sister to the parasitic trypanosomatids, leading to the conclusion that there might be free-living, parasitic kinetoplastids in Danish lake sediments. However, their phylogenetic trees had no representative sequence of $B$. saltans, used one very long-branch outgroup (Euglena gracilis) and no gamma correction. It is therefore very likely that their 'free-living parasitic Kinetoplastida' may simply be B. saltans, which sometimes 
groups as sister to the trypanosomatids [see also Simpson et al. (2002)]. It was not made clear whether their 27 different clones were all from one library or from several and, therefore, whether this number of clones per library is significantly higher than we found in our 16 libraries (only one to six different genotypes were recovered from each library; see Table 2). It is also possible that their standard PCR methods may have increased chimaera formation and therefore presented a deceptively high diversity, a problem that has been underestimated in studies of eukaryotic diversity to date (Berney et al., 2004; Cavalier-Smith, 2004).

\section{Freshwater and marine diversification in $\mathbf{N}$. designis and other bodonids}

Fig. 4. shows that morphotypes of $N$. designis fall into exclusively marine or freshwater clades; there are seven to nine freshwater clades (two being from environmental DNA only, so $N$. designis morphology was not confirmed) and one major marine clade, plus two further small, but clearly distinct, marine clades (one containing Cruzella) that mainly include environmental sequences of unknown morphology (but might, when characterized, prove to be like $N$. designis). The tree topology of clade $1 \mathrm{C}$ and the distribution of marine and freshwater strains across it suggest strongly that the ancestral $N$. designis morphotype was originally a freshwater organism and that there were only three effective colonizations of the sea: one by the major marine $N$. designis clade, one by the clade containing Cruzella and one by the unique lineage simply labelled 'kinetoplastid flagellate'. This suggests that the vast majority of strains of $N$. designis at least are not tolerant to a wide variety of environmental conditions, contrary to previous suggestions (Arndt et al., 2000; Atkins et al., 2000), but that each strain is restricted to either freshwater or marine environments. As clade C largely comprises $N$. designis morphotypes, it is clear that Cruzella evolved from such an ancestor. A separate Bayesian analysis of the Fig. 4 dataset showed exactly the same overall topology, except for clades $\mathrm{Nd} 3$ and $\mathrm{Nd} 4$ being weak sisters [posterior probability $(\mathrm{PP})=0 \cdot 72]$ and clades $1 \mathrm{D}$ and $1 \mathrm{~F}$ being sisters, even more weakly $(\mathrm{PP}=0 \cdot 5)$. On that Bayesian tree (not shown), holophyly of the major marine clade was supported moderately well $(\mathrm{PP}=0 \cdot 86)$. Holophyly of clades $\mathrm{Nd} 1$, $\mathrm{Nd} 2$ and $\mathrm{Nd} 3$, and the sister relationship of $\mathrm{Nd} 1$ and $\mathrm{Nd} 2$, were supported strongly $(\mathrm{PP}=1 \cdot 0)$. In addition, two nodes within the basal freshwater part of $\mathrm{Nd} 2$ received PP support of $1 \cdot 0$ and another received support of 0.99 . Thus, the Bayesian analysis also fully supported the derived nature of all marine Neobodo clades.

It is important to make a crude estimate of the age of clade $\mathrm{C}$ but, unfortunately, there is almost no fossil record for kinetoplastids or other excavate protozoa. However, from molecular tree topology and proportions, it would appear that excavates in general and Euglenozoa in particular arose early in the evolution of bikont eukaryotes. Assuming that the primary eukaryotic divergence was indeed between unikonts and bikonts (Stechmann \& Cavalier-Smith, 2003), it is probable that bikonts are at least 800 million years old, as eukaryotes are at least that old (Cavalier-Smith, 2002; Porter \& Knoll, 2000). Given the difficulty of resolving the branching order of the three main euglenozoan groups, it is likely that kinetoplastids diverged from euglenoids and diplonemids at fairly similar times early in the diversification of the Euglenozoa. It would therefore be surprising if the separation of Kinetoplastea was much younger than about 600 million years. As the basal kinetoplastid is significantly later than the basal node for Euglenozoa, applying a crude molecular clock to Fig. 1 in the study by von der Heyden et al. (2004b) yields a rough estimate of 360 million years for the age of the kinetoplastid cenancestor. As the depth of clade C of Fig. 3 and in Fig. 4 of von der Heyden et al. (2004b) is roughly one-tenth that of the whole kinetoplastid clade, applying a crude clock gives a rough estimate of 36 million years for the Neobodo morphotype. Another way of making an estimate would be to assume that the early Cretaceous Paleoleishmania in amber (Poinar \& Poinar, 2004) really refers to a single species and is also identified correctly as a trypanosomatid, which it may be, but need not be: how can one really rule out a mixture of other symbionts? As the Neobodo clade is internally markedly deeper on all our rRNA trees than the trypanosomatid clade, this would imply that Neobodo is older than 100 million years (the minimum age of the amber fossils) if this molecule were clock-like. However, if trypanosomatids are sisters of core bodonids, as in Fig. 4 of von der Heyden et al. (2004b), it would appear from that tree that $18 \mathrm{~S}$ rRNA has been evolving about twice as fast in core bodonids as in trypanosomatids since they diverged from their common ancestor, so a minimum figure of approximately 50 million years would be a more reasonable estimate for the age of Neobodo. Although it is hazardous to estimate ages from molecular trees, as the rRNA gene is an appallingly unreliable 'clock' (CavalierSmith, 2002, 2004), it is unlikely that an estimate of 35-50 million years old would be in error by more than two- or threefold. Thus, for tens of millions of years, the ancestrally freshwater $N$. designis complex probably only colonized the sea successfully about three times, although there may be other, as yet undiscovered marine Neobodo lineages. From Fig. 4 , the depth of the major marine clade appears to be at least one-quarter that of the Neobodo clade, suggesting that this major invasion of the marine habitat took place over 9 million years ago and no others have occurred since. The depth of the B. saltans clade is only about one-third that of Neobodo but, as the overall branch lengths of the corresponding part of the tree are similarly different, this probably reflects differences in the rate of rRNA evolution, rather than a significantly different age. Yet B. saltans is essentially always freshwater [although we isolated one strain from decaying seaweed on a beach (von der Heyden et al., 2004b) and B. saltans was reported by Vørs (1992) in the Gulf of Finland].

There is other evidence supporting our conclusion that effective interchange between freshwater and marine 
habitats is very rare in bodonids. Consider the culture experiments of Mylnikov \& Gräfenhan (reviewed by Arndt et al., 2000) and Ekelund (2002). The former transferred marine and freshwater $B$. saltans and $N$. designis and other protists gradually into less/more-saline conditions and found that they adapted to and grew in these. Both $B$. saltans and $N$. designis were transferred successfully into the other types of medium. Other bodonids ( $R$. nasuta, Parabodo nitrophilus, Parabodo caudatus) were able to withstand some, but not a total, change in salinity. Ekelund (2002) carried out similar experiments using 11 soil flagellates (including $R$. nasuta, P. caudatus, B. saltans and $N$. designis) and showed that none of the flagellates thrived in 15 parts per thousand (p.p.t.) $\mathrm{NaCl}$. In contrast to Mylnikov \& Gräfenhan (reviewed by Arndt et al., 2000), whose cultures may have been genetically mixed, Ekelund (2002) used clonal cultures and found that freshwater $N$. designis only tolerated an increased salinity up to 3 p.p.t., even when transferred gradually into higher-salinity media. Our demonstration of the great genetic diversity of morphologically indistinguishable Neobodo and B. saltans shows that physiological experiments on non-clonal cultures of bodonids (or any other protist) are ecologically meaningless. Experiments where flagellates are transferred gradually into higher/lower-salinity media also may not realistically represent many environmental situations where microbes are naturally dispersed among habitats. There are very few cases in which a small pool of sea water reduces salinity gradually every few days, thereby allowing its inhabitants to adapt slowly to the changing conditions, and then becomes freshwater. Although rivers perpetually discharging into the sea will often put freshwater microbes into gradually increasing salinity, microbes dispersed by wind, precipitation and/or animal vectors will often land in either an environment that they are capable of living in or one in which they will probably perish and not be able to compete with strains that have adapted genetically over millions of years to that habitat.

The freshwater/marine divergence is particularly prominent among strains of $N$. designis, probably because we have sufficient samples to demonstrate this. Although other bodonids do not show such clear-cut divisions between freshwater and marine lineages, the deep clade 1D that is sister to Cryptaulaxoides-like, as well as the novel bodonid lineage $1 \mathrm{E}$, each have distinct marine and freshwater subclades (Fig. 4). Within Rhynchobodo/Klosteria, marine and freshwater/soil strains group separately and there is a well-supported clade comprising strains from soil and tree moss (Fig. 3). However, within the genera Rhynchomonas, Parabodo and Procryptobia, marine and freshwater samples intermingle freely with each other; within Rhynchomonas, a strain (BSZ1) from an Atlantic deep-sea hydrothermal vent even groups with an environmental sequence (L6.8) from the Panamanian rainforest, albeit rather weakly! These apparently mixed groupings may, however, be artefacts of undersampling (similar to what appeared to be true for Neobodo when we only had a few strains), hiding ecological specialization; much more work is needed to explore the diversity and distribution of all these clades.

We showed previously that, in the cryptomonad genus Goniomonas, there is an even more fundamental divergence between marine and freshwater strains (von der Heyden et al., 2004a) and that there are also distinct marine and freshwater clades within centrohelid heliozoa (von der Heyden, 2004). Lee \& Patterson (1998), using cluster analysis on diverse protistan community assemblages, showed that communities that are geographically close together do not cluster together, but that that those from similar habitats do, and that there is little overlap in community assemblage between marine and freshwater sites. This further strengthens the hypothesis that not all protists are able to move freely between these types of habitats, but are instead restricted to a physiological/ ecological range of variables.

Our results also show that strains of $N$. designis that diverged tens of millions of years ago can coexist at the same site: we isolated several strains of $N$. designis from the same geographical area at the same time (four strains from sediment in Priest Pot, Cumbria, UK, and TeToto Gorge, New Zealand; marked on Fig. 4 with one and two dots, respectively) that actually group in very different positions on the tree. It is an open question whether this coexistence between very distinct strains reflects differences in ecological adaptations among the strains; however, given their very deep divergence on the rRNA tree, it would be surprising if they do not also differ in hundreds of protein-coding genes and we suspect that at least some such differences are likely to be adaptive. We also suspect that thin-section ultrastructural studies might reveal morphological differences among the strains. Even more detailed light microscopy would probably reveal at least some quantitative differences; previously, we noted that one freshwater strain, Neobodo sp. Cape Town (CPT; von der Heyden et al., 2004b), appeared to be morphologically distinct from typical $N$. designis in having a longer anterior cilium and an anteriorly more pointed, squatter and smaller body. However, it died before we were able to take measurements or record these differences photographically. Although some bodonids [B. saltans (Brooker, 1971), Cephalothamnium (Vickerman, 1990) and Rhynchomonas (Swale, 1973)] have ciliary hairs (mastigonemes), we found none in either Neobodo strain, confirming the observations of Eyden (1977). If the classification of Cephalothamnium is correct (Vickerman, 1990), at least one member of each of the three bodonid suborders would have ciliary hairs; possibly, therefore, their absence in $N$. designis is a secondary loss.

\section{Huge genetic variation and 'species' number of bodonids}

The degree of genetic variation within many bodonid clades is comparatively greater than that of whole orders of higher eukaryotes. Within $N$. designis, no $18 \mathrm{~S}$ rRNA gene sequences yet found are identical; this degree of variation in the $18 \mathrm{~S}$ 
rRNA gene of higher eukaryotes would correspond to separate species. Throughout the protist world, morphospecies are now being found to comprise many cryptic species complexes. Cryptic species, such as the majority of bodonids, have also been reported within Cercozoa (Kühn et al., 2000), Foraminifera (de Vargas et al., 1999; Kucera \& Darling, 2002; Pawlowski \& Holzmann, 2002), the spumellarid radiozoan Thalassicolla nucleata (Zettler et al., 1998), ciliates (Nanney et al., 1989, 1998; Fokin et al., 2004), dinoflagellates (John et al., 2003) and certain types of picoplankton (Potter et al., 1997). The almost-universal presence of such cryptic variation makes the use of the morphospecies in discussions of protist biodiversity and dispersal (Finlay, 2002; Finlay et al., 1996) of limited value as, in bodonids and many other protists, there is no reason to think that named morphospecies have much real biological significance. The conventional 'species' boundaries may largely reflect our ignorance and current inability to subdivide them into more biologically meaningful units.

Cryptic speciation in all lineages and the relatively long evolutionary history of free-living kinetoplastids have produced a much larger number of species than realized previously. Although there are descriptions of over 200 nominal species, many have not been based on pure cultures and are practically unrecognizable; there may be different stages of the life cycle of single species and, hence, the actual number of morphotypes may have been overestimated (Zhukov, 1991). Recent protozoologists can confidently recognize only about 15 'species' within 11 genera. For example, the three 'species' of Rhynchomonas [R. nasuta, Rhynchomonas mutabilis and Rhynchomonas metabolita, reviewed by Zhukov (1971)] are currently all attributed to only one species, $R$. nasuta, as the only real differences between them were in cell size and it is possible that there are physiological or ecological species within the morphological species' (Zhukov, 1991). The molecular evidence presented here indicates that many of the currently recognizable morphotypes may contain hundreds of distinct lineages; much further work is needed to determine whether each of them represents a different evolutionary peak adapted to particular habitat features, be these the type of prey utilized, the amount of moisture in the surroundings, sediment/soil-grain size or coarseness or other environmental variables. Recent experiments by Smirnov \& Thar (2003) studied the microspatial distribution of amoebae and confirmed the presence of microhabitats, which were occupied selectively by amoebic species; similar ecological work is needed for the bodonid cryptic 'species'. In contrast to ciliates, where breeding experiments have shown that there are numerous cryptic biological species within a common morphotype (Fokin et al., 2004; Nanney et al., 1989, 1998), it is not known whether most bodonids are sexual or asexual, so we have no idea whether the biological species concept applies to them. Nanney et al. $(1989,1998)$ suggested that 'genetically divergent tetrahymenids are morphologically identical because of stabilizing selection that preserves a morphological phenotype which probably represents some adaptive peak'. We predict that future studies will reveal important ecological and physiological differences among the lineages comprising rather ancient morphospecies, such as B. saltans, N. designis and R. nasuta, and that, one day, they will be divided into numerous 'species'. The fact that they are currently morphologically indistinguishable does not mean that they are really morphologically identical. Detailed electron microscopy or sophisticated light microscopy with special staining methods might possibly reveal significant morphological differences.

\section{Are bodonids really all cosmopolitan?}

von der Heyden et al. (2004b) showed some evidence for a restricted biogeographical distribution of strains from the northern and southern hemispheres in clade 3 (the B. saltans species complex). Addition of 10 environmental sequences confirms that the four clades recovered previously are still present and fall into northern-hemisphere, southern-hemisphere and two clades of a mixture of hemispheres (Fig. 9), suggesting that some strains of $B$. saltans may have a restricted biogeographical distribution and are not ubiquitously distributed globally. This conclusion must still be tentative because much more sampling is needed for a decisive test. However, in certain exceptionally well-sampled morphospecies of pelagic Foraminifera, the study of the geographical distribution of genotypes with distinctive rRNA genes shows that some are not cosmopolitan, as was assumed incorrectly when only morphospecies were studied (Darling et al., 2004). In the very well-sampled Paramecium aurelia species complex, some biological species are cosmopolitan, but others clearly are not (Fokin et al., 2004). In the Alexandrium tamarense complex, there also appear to be geographically restricted clades (John et al., 2003). Thus, allopatric speciation in protists may not be as rare as sometimes claimed (Finlay \& Fenchel, 2004). As Patterson \& Lee (2000) and Coleman (2002) pointed out and Finlay \& Fenchel $(1999,2004)$ also recognized, if morphospecies are seriously undersplit taxonomically, this can inflate the appearance of cosmopolitanism substantially. Our genetic studies of bodonids indicate strongly that many morphospecies have been grossly undersplit taxonomically and show that fine genetic discrimination, combined with even more extensive sampling, must be a central feature of future biogeographical studies of zooflagellates. We suspect that such studies will show that at least some bodonid genotypes are indeed cosmopolitan, as predicted by Finlay \& Fenchel $(1999,2004)$ and Finlay et al. (2004), but will reveal others with lower dispersal/colonization potential that are not.

\section{Clade-specific analyses of freshwater and marine neobodonids}

The genetic diversity found for a part of freshwater $N$. designis subclade 1 by using specific PCR primers (marked by a box on Fig. 4) was somewhat greater than was retrieved for primers that sampled across a whole group [see Bass \& 
Cavalier-Smith (2004) for discussion of this]. We found that, even for very closely related sequences (i.e. FW3.3/ FW12.5 or FW18.4/FW20.3), there is at least a difference of $2 \mathrm{nt}$ in this highly variable region of the $18 \mathrm{~S}$ rRNA gene sequence and that, over the whole length of the gene, this difference is probably even greater. This suggests that there are probably hundreds of individual lineages, even within this very narrow subclade of $N$. designis. Clearly, much more sampling of all bodonid lineages (and probably all protozoan lineages) is required in order to ascertain their true global diversity, as we did not retrieve the same sequence from different parts of the globe. Although we did not find any confirming instance of cosmopolitanism for even one genotype, there is little evidence of endemicity or restricted biogeography within individual clades. We found one clade containing sequences exclusively from New Zealand, two exclusively from the UK and one exclusively from Priest Pot (Cumbria, UK); all other clusters of sequences are from very different geographical areas (Fig. 7).

Within the marine clade are four subclades (Fig. 8; labelled M1-M4); two of these (M2 and M3) are composed entirely of sequences from either the northern or southern hemisphere; M4 contains only one environmental sequence from Panama and M1 contains a sequence from a deep-sea hydrothermal vent (9-AT5), an environmental sequence from New Zealand and an N. designis isolate from Denmark (Fig. 8). Thus, N. designis, like B. saltans, shows several clades that are suggestive of restricted biogeographical distribution. These would merit more intensive sampling to determine whether this prima facie evidence of endemicity is genuine, as similar evidence appears to be for some foraminifera, or simply a consequence of the presently unavoidable undersampling of their immense genotypic diversity.

\section{ACKNOWLEDGEMENTS}

S.v.d.H. thanks the Natural Environment Research Council UK (NERC; Marine and Freshwater Microbial Biodiversity Programme) for financial support, Professor K. Vickerman for donation of Dimastigella strains, Dr C. J. von der Heyden and $\mathrm{Mr}$ and Mrs Holdstock for sample collections, Professor D. Hamilton for sample collection in New Zealand, Mr K. Clarke for electron microscopy of neobodonid strains, Miss A. Williams for help with light microscopy, Dr T. Stoeck for sharing pre-published results and Bland Finlay (CEH) for help with sample collection and valuable discussion. T. C.-S. thanks NERC for research grants, the Canadian Institute for Advanced Research and NERC for fellowship support.

\section{REFERENCES}

Al-Qassab, S., Lee, W. J., Murray, S., Simpson, A. G. B. \& Patterson, D. J. (2002). Flagellates from stromatolites and surrounding sediments in Shark Bay, Western Australia. Acta Protozool 41, 91-144.

Altschul, S. F., Gish, W., Miller, W., Myers, E. W. \& Lipman, D. J. (1990). Basic local alignment search tool. J Mol Biol 215, 403-410.
Arndt, H., Dietrich, D., Auer, B., Cleven, E.-J., Gräfenhan, T., Weitere, M. \& Mylnikov, A. P. (2000). Functional diversity of heterotrophic flagellates in aquatic ecosystems. In The Flagellates: Unity, Diversity and Evolution, pp. 240-268. Edited by B. S. C. Leadbeater \& J. C. Green. London: Taylor \& Francis.

Atkins, M. S., Anderson, O. R. \& Wirsen, C. O. (1998). Effect of hydrostatic pressure on the growth rates and encystment of flagellated protozoa isolated from a deep-sea hydrothermal vent and a deep shelf region. Mar Ecol Prog Ser 171, 85-95.

Atkins, M. S., Teske, A. P. \& Anderson, O. R. (2000). A survey of flagellate diversity at four deep-sea hydrothermal vents in the Eastern Pacific Ocean using structural and molecular approaches. J Eukaryot Microbiol 47, 400-411.

Bass, D. \& Cavalier-Smith, T. (2004). Phylum-specific environmental DNA analysis reveals remarkably high global biodiversity of Cercozoa (Protozoa). Int J Syst Evol Microbiol 54, 2393-2404.

Berney, C., Fahrni, J. \& Pawlowski, J. (2004). How many novel eukaryotic 'kingdoms'? Pitfalls and limitations of environmental DNA surveys. BMC Biol 2, 13. http://www.biomedcentral.com/17417007/2/13

Brooker, B. E. (1971). Fine structure of Bodo saltans and Bodo caudatus (Zoomastigophora, Protozoa) and their affinities with the Trypanosomatidae. Bull Brit Mus Nat Hist 22, 89-102.

Callahan, H. A., Litaker, R. W. \& Noga, E. J. (2002). Molecular taxonomy of the suborder Bodonina (order Kinetoplastida), including the important fish parasite, Ichthyobodo necator. I Eukaryot Microbiol 49, 119-128.

Cavalier-Smith, T. (1981). Eukaryote kingdoms: seven or nine? Biosystems 14, 461-481.

Cavalier-Smith, T. (2002). The neomuran origin of archaebacteria, the negibacterial root of the universal tree and bacterial megaclassification. Int J Syst Evol Microbiol 52, 7-76.

Cavalier-Smith, T. (2003a). Protist phylogeny and the high-level classification of Protozoa. Eur J Protistol 39, 338-348.

Cavalier-Smith, T. (2003b). The excavate protozoan phyla Metamonada Grassé emend. (Anaeromonadea, Parabasalia, Carpediemonas, Eopharyngia) and Loukozoa emend. (Jakobea, Malawimonas): their evolutionary affinities and new higher taxa. Int J Syst Evol Microbiol 53, 1741-1758.

Cavalier-Smith, T. (2004). Only six kingdoms of life. Proc R Soc Lond B Biol Sci 271, 1251-1262.

Coleman, A. W. (2002). Microbial eukaryote species. Science 297, 337.

Darling, K. F., Kucera, M., Pudsey, C. J. \& Wade, C. M. (2004). Molecular evidence links cryptic diversification in polar planktonic protists to Quaternary climate dynamics. Proc Natl Acad Sci U S A 101, 7657-7662.

de Vargas, C., Norris, R., Zaninetti, L., Gibb, S. W. \& Pawlowski, J. (1999). Molecular evidence of cryptic speciation in planktonic foraminifers and their relation to oceanic provinces. Proc Natl Acad Sci U S A 96, 2864-2868.

Doležel, D., Jirků, M., Maslov, D. A. \& Lukeš, J. (2000). Phylogeny of the bodonid flagellates (Kinetoplastida) based on small-subunit rRNA gene sequences. Int J Syst Evol Microbiol 50, 1943-1951.

Ekelund, F. (2002). Tolerance of soil flagellates to increased $\mathrm{NaCl}$ levels. J Eukaryot Microbiol 49, 324-328.

Eyden, B. P. (1977). Morphology and ultrastructure of Bodo designis Skuja 1948. Protistologica 13, 169-179.

Finlay, B. J. (2002). Global dispersal of free-living microbial eukaryote species. Science 296, 1061-1063. 
Finlay, B. J. \& Fenchel, T. (1999). Divergent perspectives on protist species richness. Protist 150, 229-233.

Finlay, B. J. \& Fenchel, T. (2004). Cosmopolitan metapopulations of free-living microbial eukaryotes. Protist 155, 237-244.

Finlay, B. J., Corliss, J. O., Esteban, G. \& Fenchel, T. (1996). Biodiversity at the microbial level: the number of free-living ciliates in the biosphere. Q Rev Biol 71, 221-237.

Finlay, B. J., Esteban, G. F. \& Fenchel, T. (2004). Protist diversity is different? Protist 155, 15-22.

Fokin, S. I., Przyboś, E., Chivilev, S. M., Beier, C. L., Horn, M., Skotarczak, B., Wodecka, B. \& Fujishima, M. (2004). Morphological and molecular investigations of Paramecium schewiakoffi sp. nov. (Ciliophora, Oligohymenophorea) and current status of distribution and taxonomy of Paramecium spp. Eur J Protistol 40, 225-243.

Hall, T. A. (1999). BioEdit: a user-friendly biological sequence alignment editor and analysis program for Windows 95/98/NT. Nucleic Acids Symp Ser 41, 95-98.

Huber, T., Faulkner, G. \& Hugenholtz, P. (2004). BELLEROPHON; a program to detect chimeric sequences in multiple sequence alignments. Bioinformatics 20, 2317-2319.

John, U., Fensome, R. A. \& Medlin, L. K. (2003). The application of a molecular clock based on molecular sequences and the fossil record to explain biogeographic distributions within the Alexandrium tamarense "species complex" (Dinophyceae). Mol Biol Evol 20, $1015-1027$.

Kucera, M. \& Darling, K. F. (2002). Cryptic species of planktonic foraminifera: their effect on palaeoceanographic reconstructions. Philos Trans $R$ Soc Lond A 360, 695-718.

Kühn, S., Lange, M. \& Medlin, L. K. (2000). Phylogenetic position of Cryothecomonas inferred from nuclear-encoded small subunit ribsomal RNA. Protist 151, 337-345.

Larsen, J. \& Patterson, D. J. (1990). Some flagellates (Protista) from tropical marine sediments. J Nat Hist 24, 801-937.

Lee, W. J. \& Patterson, D. J. (1998). Diversity and geographic distribution of free-living heterotrophic flagellates - analysis by PRIMER. Protist 149, 229-244.

López-García, P., Philippe, H., Gail, F. \& Moreira, D. (2003). Autochthonous eukaryotic diversity in hydrothermal sediment and experimental microcolonizers at the Mid-Atlantic Ridge. Proc Natl Acad Sci U S A 100, 697-702.

Moon-van der Staay, S. Y., De Wachter, R. \& Vaulot, D. (2001). Oceanic $18 \mathrm{~S}$ rDNA sequences from picoplankton reveal unsuspected eukaryotic diversity. Nature 409, 607-610.

Moreira, D. \& López-Garcia, P. (2002). The molecular ecology of microbial eukaryotes unveils a hidden world. Trends Microbiol 10, 31-38.

Moreira, D. \& López-García, P. (2003). Are hydrothermal vents oases for parasitic protists? Trends Parasitol 19, 556-558.

Moreira, D., López-García, P. \& Vickerman, K. (2004). An updated view of kinetoplastid phylogeny using environmental sequences and a closer outgroup: proposal for a new classification of the class Kinetoplastea. Int J Syst Evol Microbiol 54, 1861-1875.

Nanney, D. L., Meyer, E. B., Simon, E. M. \& Preparata, R. M. (1989). Comparison of ribosomal and isozymic phylogenies of tetrahymenine ciliates. J Protozool 36, 1-8.

Nanney, D. L., Park, C., Preparata, R. \& Simon, E. M. (1998), Comparison of sequence differences in a variable $23 \mathrm{~S}$ rRNA domain among sets of cryptic species of ciliated protozoa. J Eukaryot Microbiol 45, 91-100.

Patterson, D. J. \& Lee, W. J. (2000). Geographic distribution and diversity of free-living heterotrophic flagellates. In The Flagellates:
Unity, Diversity and Evolution, pp. 269-287. Edited by B. S. C. Leadbeater \& J. C. Green. London: Taylor \& Francis.

Patterson, D. J. \& Simpson, A. G. B. (1996). Heterotrophic flagellates from coastal marine and hypersaline sediments in Western Australia. Eur J Protistol 32, 423-448.

Pawlowski, J. \& Holzmann, M. (2002). Molecular phylogeny of foraminifera: a review. Eur J Protistol 38, 1-10.

Poinar, G., Jr \& Poinar, R. (2004). Paleoleishmania proterus n. gen., n. sp., (Trypanosomatidae: Kinetoplastida) from Cretaceous Burmese amber. Protist 155, 305-310.

Porter, S. M. \& Knoll, A. H. (2000). Testate amoebae in the Neoproterozoic Era: evidence from vase-shaped microfossils in the Chuar Group, Grand Canyon. Paleobiology 26, 360-385.

Posada, D. \& Crandall, K. A. (1998). MODELTEST: testing the model of DNA substitution. Bioinformatics 9, 817-818.

Potter, D., Lajeunesse, T. C., Saunders, G. W. \& Anderson, R. A. (1997). Convergent evolution masks extensive biodiversity among marine coccoid picoplankton. Biodivers Conserv 6, 99-107.

Rasmussen, L. D., Ekelund, F., Hansen, L. H., Sørensen, S. J. \& Johnsen, K. (2001). Group-specific PCR primers to amplify 24S $\alpha$-subunit rRNA genes from Kinetoplastida (Protozoa) used in denaturing gradient gel electrophoresis. Microb Ecol 42, 109-115.

Robison-Cox, J. F., Bateson, M. M. \& Ward, D. M. (1995). Evaluation of nearest-neighbor methods for detection of chimeric small-subunit rRNA sequences. Appl Environ Microbiol 61, 1240 1245 .

Simpson, A. G. B., Lukeš, J. \& Roger, A. J. (2002). The evolutionary history of kinetoplastids and their kinetoplasts. Mol Biol Evol 19, 2071-2083.

Skuja, H. (1948). Taxonomie des Phytoplanktons einiger Seen in Uppland, Schweden. Symb Bot Upsal 9, 1-399 (in German).

Smirnov, A. \& Thar, R. (2003). Spatial distribution of Gymnamoebae (Rhizopoda, Lobosea) in brackish-water sediments at the scale of centimeters and millimeters. Protist 154, 359-369.

Stechmann, A. \& Cavalier-Smith, T. (2003). The root of the eukaryote tree pinpointed. Curr Biol 13, R665-R666.

Swale, E. M. F. (1973). A study of the colourless flagellate Rhynchomonas nasuta (Stokes) Klebs. Biol J Linn Soc 5, 255-264.

Swofford, D. L. (2001). PAUP*: Phylogenetic Analysis Using Parsimony (*and other methods), v. 4.0b6. Sunderland, MA: Sinauer Associates.

UKNCC (2001). Catalogue of the UK National Culture Collection - List of Algae and Protozoa. Oban, UK: United Kingdom National Culture Collection.

van Hannen, E. J., Mooij, W., van Agterveld, M. P., Gons, H. J. \& Laanbroek, H. J. (1999). Detritus-dependent development of the microbial community in an experimental system: qualitative analysis by denaturing gradient gel electrophoresis. Appl Environ Microbiol 65, 2478-2484.

Vickerman, K. (1990). Phylum Zoomastigina, class Kinetoplastida. In Handbook of Protoctista, pp. 215-238. Edited by L. Margulis, J. O. Corliss, M. Melkonian \& D. J. Chapman. Boston: Jones \& Bartlett.

von der Heyden, S. (2004). Testing ubiquitous dispersal and freshwater/marine divergence within free-living protist groups. DPhil thesis, University of Oxford.

von der Heyden, S., Chao, E. E. \& Cavalier-Smith, T. (2004a). Genetic diversity of goniomonads: an ancient divergence between marine and freshwater species. Eur J Phycol 39, 343-350. 
von der Heyden, S., Chao, E. E., Vickerman, K. \& Cavalier-Smith, T. (2004b). Ribosomal RNA phylogeny of bodonid and diplonemid flagellates and the evolution of Euglenozoa. J Eukaryot Microbiol 51, 402-416.

Vørs, N. (1992). Heterotrophic amoebae, flagellates and heliozoa from the Tvärminne area, Gulf of Finland, in 1988-1990. Ophelia 36, 1-109.

Vørs, N. (1993). Marine heterotrophic amoebae, flagellates and heliozoa from Belize (Central America) and Tenerife (Canary Islands), with descriptions of new species, Luffisphaera bulbochaete N. Sp., L. longihastis N. Sp., L. turriformis N. Sp. and Paulinella intermedia N. Sp. J Eukaryot Microbiol 40, 272-287.
Zettler, L. A. A., Anderson, O. R. \& Caron, D. A. (1998). Insights on the diversity within a "species" of Thalassicolla (Spumellarida) based on 16S-like ribosomal RNA gene sequencing. J Eukaryot Microbiol 45, 488-496.

Zhukov, B. F. (1971). A key to colourless free-living flagellates of the suborder Bodonina Hollande. In Biology and Productivity of Freshwater Organisms, pp. 241-283. Edited by M. M. Kamshilov. Leningrad: Science Press (in Russian).

Zhukov, B. F. (1991). The diversity of bodonids. In The Biology of Free-living Heterotrophic Flagellates, pp. 177-184. Edited by D. J. Patterson \& J. Larsen. Oxford: Clarendon Press. 